\title{
Análisis de las competencias en la educación superior a través de flipped classroom
}

\section{Analysis of competencies in Higher Education through Flipped Classroom}

\author{
Emilio Abad-Segura ${ }^{1}$ (이 @ ; Mariana Daniela González-Zamar ${ }^{1}$ 이 @ \\ ${ }^{1}$ Universidad de Almería (UAL), España.
}

\begin{abstract}
Resumen
El proceso de enseñanza-aprendizaje en la universidad exige una continua adaptación a las nuevas propuestas metodológicas. Las clases magistrales han dado paso a estudiantes activos, abiertos a desarrollar las competencias necesarias para su futuro profesional. El modelo flipped classroom adquiere mayor importancia en las aulas universitarias por su enfoque constructivista, trasladando el foco del profesor al alumno. Se describen algunos de los aspectos más relevantes de este modelo de aprendizaje a partir de una revisión bibliográfica. Los resultados están en línea con la búsqueda de capacidades como la autonomía, el análisis interpretativo y la autocrítica.

Palabras clave: flipped classroom; aula invertida; aprendizaje; educación superior; aula universitaria; métodos de enseñanza; docencia universitaria.
\end{abstract}

\begin{abstract}
The teaching-learning process in the university requires a continuous adaptation to the new methodological proposals. The master classes have given way to active students, open to developing the necessary skills for their professional future. The flipped classroom model acquires greater importance in the university classrooms because of its constructivist approach, transferring the teacher's focus to the student. Some of the most relevant aspects of this learning model are described based on a literature review. The results are in line with the search for skills such as autonomy, interpretative analysis and self-criticism.

Keywords: flipped classroom; learning; higher education; university classroom; teaching methods; university teaching.
\end{abstract}

\section{Introducción}

La puesta en marcha del Espacio Europeo de Enseñanza Superior (EEES) en 2008 y su consiguiente propuesta de renovación metodológica a nivel didáctico, han propiciado el desarrollo de nuevos modelos metodológicos por parte de los docentes universitarios, con las Tecnologías de la Información y la Comunicación (TIC) como eje central de muchas de estas propuestas.

Por otro lado, el EEES incluye la evaluación por competencias. Entre sus objetivos principales se encuentra la empleabilidad, donde señala que los títulos universitarios deben ser coherentes con el principio de libre movilidad de estudiantes y titulados. La garantía de este principio es necesaria para la construcción del EEES al tiempo que se constituye como un pilar básico del derecho comunitario, contenido tanto en los Tratados Fundacionales como en el derecho derivado. De este modo, los 
planes de estudios universitarios deben preparar a los estudiantes al acceso del ejercicio profesional, con el objetivo de mejorar la empleabilidad de los titulados (Herreid y Schiller, 2013).

Las bases pedagógicas que sustentan el modelo, la taxonomía de Bloom, el cono de aprendizaje de Edgar Dale y el constructivismo social, relacionan que con el modelo invertido, en contra del modelo tradicional, en la clase los estudiantes pueden aplicar, analizar, evaluar y crear contenido en torno a un tema determinado, mientras que las tareas de memorizar y comprender se realizan fuera del aula. Es decir, la aplicación práctica del contenido, como momento más relevante del proceso de proceso de aprendizaje, se realiza en la clase con el docente como guía y con el resto de compañeros (Andrade y Chacón, 2018; Manresa, 2018).

En esta línea, también se fomenta que los alumnos universitarios realicen estancias en otros países de Europa como parte de su educación formal, desarrollando habilidades, actitudes y valores útiles para su futuro profesional. Es importante comprender el marco teórico donde se desarrollan los nuevos profesionales y las competencias que deben adquirir para poder diseñar un espacio de enseñanza óptimo.

30 Así, se debe preparar a las próximas generaciones en la adquisición de conocimientos y competencias para afrontar el futuro mundo laboral impreciso, además de proporcionarles herramientas para ser ciudadanos que participen en una sociedad democrática asumiendo sus responsabilidades personales y colectivas (Huber, 2008). En esta revolución que afecta de lleno a las universidades, las dudas y cuestionamientos relativos a las nuevas exigencias y roles que asumen sus protagonistas, profesores y alumnos, adquiere gran relevancia (Phillips y Trainor, 2014; Thai, De Wever y Valcke, 2017).

La aplicación de nuevas metodologías que incluyan componentes didácticos que aporten y contribuyan al cumplimiento de los retos y propósitos planteados por el EEES, ya no será por parte de los profesores una opción sino una obligación, es decir, son unas herramientas que deberán incorporar o complementar a sus actuales metodologías de enseñanza.

La figura del docente universitario acostumbrado a emitir conocimientos de forma magistral adquiere una nueva perspectiva transformándose en un orientador y guía en el camino formativo académico del estudiante. Asimismo, el alumno deja de ser un receptor pasivo de contenidos, acostumbrado a recibir información y se convierte en el foco activo del aprendizaje (Díaz-Barriga, 2011). 
El espacio físico también adquiere relevancia y se modifica, ya que desaparece la clase-aula como el único escenario posible, surgiendo recursos y espacios reales y virtuales diseñados para cumplir con el objetivo de diversificación del proceso de aprendizaje. El espacio de la "red virtual" plantea un trabajo más colaborativo y activo a la hora de la búsqueda de información por parte de los alumnos aprovechando así las herramientas que nos ofrecen la tecnología (Gutiérrez, Palacios y Torrego, 2010). Es decir, hay una transformación completa y profunda; el profesor pasa de ser un proveedor de información a ser un facilitador de las fuentes de información y del aprendizaje.

Son muchas las mejoras que pueden incluirse cuando se apuesta por el cambio metodológico que propone el EEES. Entre todas las posibilidades que puedan surgir a la hora de plantear un adecuado método de enseñanza-aprendizaje, la clave está en combinar un modelo que por un lado desarrolle clases presenciales y por otro potencie el trabajo fuera del aula con ayuda de las TIC, incrementando así la flexibilidad, autorregulación, adaptación y autoaprendizaje del alumno (Cabrera, 2005). Dentro de este esquema de trabajo, nos centraremos en un método de aprendizaje activo y colaborativo del sistema pedagógico que está adquiriendo gran relevancia: flipped classroom o aula invertida.

Este método de aprendizaje supone un cambio para reducir la deserción escolar, además de mejorar el rendimiento y la motivación, e incrementar la percepción del alumno, ya que al favorecer el trabajo autónomo, colaborativo y autoaprendizaje se convierte en un método flexible y adaptable (Sánchez y Arrufat, 2016; Mason, Shuman y Cook, 2013; Touchton, 2015).

El principal objetivo de este trabajo es conocer el impacto que supone la aplicación del modelo flipped classroom en las competencias que debe adquirir un estudiante al final del proceso de aprendizaje en la educación superior.

En este artículo se analiza la influencia del aula invertida en las competencias que los estudiantes de educación superior deben desarrollar. 


\section{Modelo pedagógico flipped classroom}

Flipped classroom es un método, una estrategia y un instrumento pedagógico. Esta expresión, que en inglés se entiende como una clase al revés o dar la vuelta a la clase, consiste en que los temas que antes se hacían en clase, ahora se realizan en la casa, y a la inversa. No puede considerarse como innovación educativa, puesto que los primeros antecedentes datan de los años 90 .

Los pilares fundamentales del método plantean como premisas las cuatro letras iniciales que dieron origen al concepto FLIP en inglés: $f$ (flexible environments), I (learning culture), i (intentional content) y $p$ (professional educators) (Hamdan, Mcknight, Mcknight y Arfstrom, 2013:5).

Lage, Platt, y Treglia (2000) fueron los primeros en trabajar con el término, aunque la expresión adquirió solidez cuando, en 2007, Bergman y Sams $(2012,2014)$ grabaron una serie de vídeos para los alumnos que no podían asistir a sus clases. La sorpresa ocurrió cuando descubrieron que muchos otros estudiantes vieron estas grabaciones.

Esta metodología docente implica que la parte más importante del proceso enseñanza-aprendizaje, donde el docente controla que el análisis, la reflexión y la creación, se produzca en el aula; mientas que la lectura, presentaciones, grabaciones y la compresión se desarrollan fuera del aula (figura 1) (Bergmann y Sams, 2014, 2012; Hamdan et al., 2013: 5).
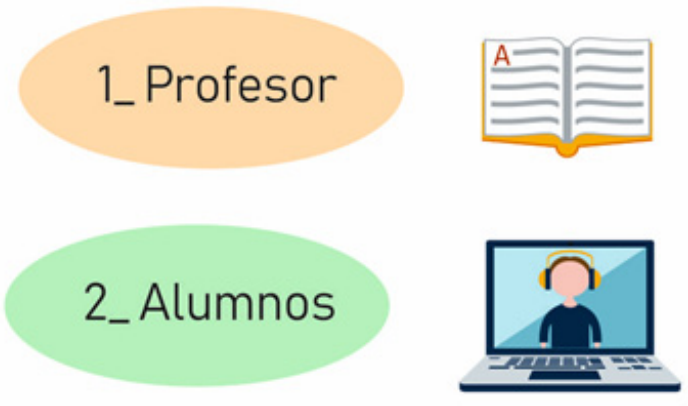

Definir objetivos de aprendizaje

Elegir el tema

Diseñar los recursos

Enviar la información

Organizar las actividades y dificultad

Visionar y leer los textos propuestos

Rellenar on line cuestionario de

seguimiento

Repasar el vídeo las veces que to necesite

Figura 1. Modelo Flipped Classroom. Antes de la clase. Fuente: Elaboración propia.

De este modo, el tiempo que se destinaba en la clase magistral a explicar conceptos y términos teóricos, se emplea a resolver dudas y cuestiones prácticas, además de favorecer la interacción y colaboración con los compañeros (figura 2). El trabajo 
conjunto entre profesor y alumno, con el material de apoyo propuesto por el primero, y el estudio, trabajo y revisión por parte del segundo, deben ser impecables para que el proceso funcione (Andrade y Chacón, 2018).

Durante la clase, los estudiantes mediante el aprendizaje cooperativo, participarán en grupos de discusión y aplicarán los conceptos clave del tema estudiado, además se debe generar una retroalimentación entre los alumnos y el docente con objeto de aclarar las posibles dudas que hayan surgido.

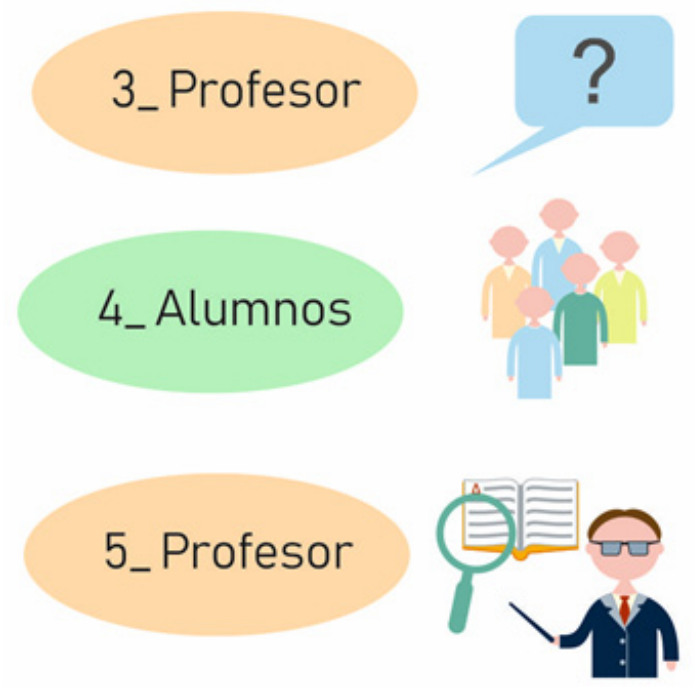

Resolver las dudas

Detectar dificultades de compresión y aprendizaje

Revisar nuevos conceptos

Adaptar los contenidos según respuestas obtenidas en los cuestionarios

Plantear actividades grupales

Fomentar el trabajo colaborativo y

cooperativo

Propiciar un aprendizaje activo

Proponer actividades de consolidación

Actuar de guía frente a los alumnos

Supervisar cómo ha sido el trabajo en casa

Figura 2. Modelo Flipped Classroom. Durante la clase. Fuente: Elaboración propia.

Así, una vez terminada la clase presencial, los estudiantes aplicarán un pensamiento crítico para analizar lo aprendido en las dos fases anteriores, además de revisar el material propuesto por el profesor (figura 3).
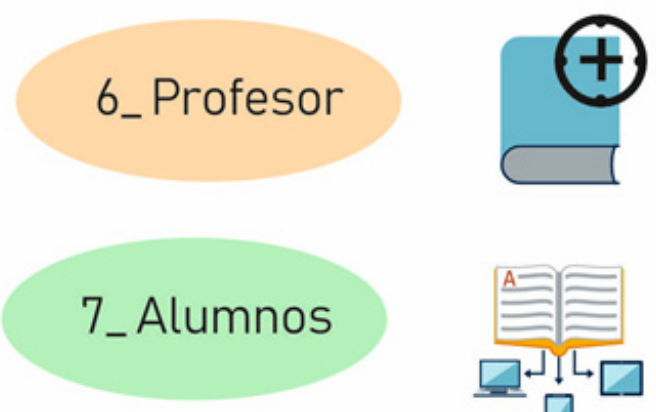

Plantear profundidad en el aprendizaje de

los conceptos adquiridos

Ofrecer explicaciones y

Brindar recursos adicionales

Repasar los nuevos conceptos

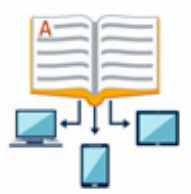

Poner en práctica las recomendaciones del profesor

Aplicar los conocimientos adquiridos Hacer uso de las herramientas de trabajo

colaborativo y cooperativo

Trabajar de forma autónoma

Figura 3. Modelo Flipped Classroom. Después de la clase.

Fuente: Elaboración propia. 


\section{Flipped classroom en la enseñanza universitaria 3.1 Aprendizaje significativo: una nueva forma de enseñar y aprender}

La teoría del aprendizaje significativo identifica cuáles son las variables que intervienen en el modelo de aprendizaje flipped classroom. Desde la óptica de la psicología y del análisis son los alumnos quienes deben asumir la responsabilidad de su aprendizaje. No se pretende que las instituciones educativas y los profesores renuncien a sus responsabilidades, sino que orienten sus objetivos a actuar de guías de aprendizaje proporcionando las herramientas y estrategias necesarias al alumnado. Así, el aprendizaje en el aula será significativo y permitirá desarrollar habilidades de autonomía, disciplina y motivación, además de aumentar el rendimiento y los resultados académicos (Abeysekera y Dawson, 2015).

La importancia de la responsabilidad y la motivación por parte del estudiante a la hora de absorber, transformar y reflexionar la información que recibe. De esta manera, el alumno reflexiona sobre su aprendizaje y lo asocia con situaciones que optimizan su experiencia educativa, apoyándose en motivaciones e intereses personales y también en problemáticas o cuestiones que requieren de solución.

Se requieren de unos principios elementales para que el aprendizaje y la experiencia en el proceso sean óptimos para el alumno:

- El aprendizaje en el aula proyecta situaciones reales y propias del entorno en el que se desarrolla.

- Las experiencias de casos reales lleven al planteamiento del problema-solución mediante la reflexión.

- El análisis de la información por parte del estudiante debe realizarse tras contar con toda la documentación y poder así valorarla, cuestionarla y comprenderla.

- El planteamiento de soluciones surgen del análisis realizado.

- La puesta en práctica y su aplicación a hechos cotidianos otorgan practicidad al proceso.

En este marco, el estudiante es el promotor de la evaluación y reflexión. Deberá plantearse preguntas adaptadas a su realidad y práctica circundante, siendo a la vez conciso al transmitir las dudas al profesor del proceso (Acaso, 2013).

La ausencia de límites en el aprendizaje y crecimiento intelectual y cognitivo del alumno es uno de los retos que plantea el aula invertida. La base autónoma del procedimiento práctico que se plantea en la resolución de un problema lo favorece. 
Las estrategias de aprendizaje por parte del profesorado para contribuir a lograr el entendimiento e interpretación integral en el acto académico, constituyen actividades conscientes e intencionales que guían las acciones para alcanzar determinadas metas de aprendizaje (Mok, 2014). Interactuar e intercambiar experiencias, aptitudes y competencias por parte de los estudiantes entre sí, les supone la posibilidad de potenciar los conocimientos potenciando y fortalecer la comunicación oral y escrita, las habilidades sociales y afianzar valores de libertad y seguridad a la hora de participar. Trabajar con una estrategia clara de aprendizaje posibilita crecer y afianzar en todo momento las actividades planteadas relacionando cada una de las fases a sus objetivos y metas planteados.

\section{Aprendizaje cooperativo: una herramienta pedagógica}

El eje que sustenta al aprendizaje cooperativo son los propios alumnos. Su protagonismo y participación constituyen piezas claves en el aprendizaje. En este sentido, numerosos autores plantean el concepto de aprendizaje cooperativo como la interacción cooperativa entre los estudiantes, en lo relativo a algún ejercicio o tema planteado por el profesor a partir del diseño o elaboración de determinadas instrucciones (Prieto, 2007a, 2007b; García, Traver y Candela, 2001; Kagan, 1994). Por otro lado, el éxito de este aprendizaje reside en el planteamiento de las actividades en pequeños grupos de alumnos (Johnson y Johnson, 1991).

El método del aprendizaje cooperativo consiste en trabajar en equipo alcanzando objetivos en común, interviniendo y asumiendo tanto responsabilidades como las consecuencias derivadas, cada uno de los integrantes del grupo. Así, todos aportan y se apropian de los conocimientos, habilidades, aptitudes y experiencias.

Flipped classroom y el aprendizaje cooperativo se encuentran asociados y complementados en el sentido de que las actividades desarrolladas antes en casa, ahora se realizan en el aula junto al resto de compañeros, permitiendo cooperar de forma grupal y transmitir al profesor las dudas generadas. El profesor deberá elaborar de forma cuidada y estructurada los contenidos que desea transmitir a su grupo mediante los recursos que considere óptimos para su comprensión. Tarea nada sencilla y que requiere de gran esfuerzo, dedicación, profundidad y estudio. 
El aprendizaje cooperativo requiere de una gran planificación por parte del profesor para llevar adelante una determinada actividad. En este sentido, se centra en el carácter autónomo del grupo de alumnos, siendo así más flexible la configuración metodológica (Zañartu, 2003).

Al combinar el aula invertida con el aprendizaje cooperativo, tratamos de eliminar el tradicional aprendizaje individual y competitivo. Ahora el beneficio personal no está por encima de los intereses y objetivos del grupo, de modo que el aprendizaje cooperativo plantea un doble propósito al adquirir conocimientos; por un lado, trabajar por el bien y beneficio común, y, por otro lado, el desarrollo individual para obtener un aprendizaje de calidad (García, Traver y Candela, 2001).

\section{Cambios en los roles asociados al profesor universitario $y$ al estudiante}

Las capacidades, habilidades y aptitudes que debe adquirir un profesor para poder llevar adelante una clase magistral son numerosas. A veces, la falta de experiencia, un nivel de conocimiento medio de la asignatura, la falta de motivación o la carencia de aptitudes comunicativas, suponen que ésta no sea la metodología adecuada.

La búsqueda de otros métodos de enseñanza como la flipped classroom, hacen posible que el profesor fomente un aprendizaje constructivo y cooperativo en su alumnado.

Poner en práctica este método no supone que el profesor pierda importancia o protagonismo en la clase, ni tampoco deduce que el profesor delegará trabajo y responsabilidad en el alumnado. Llevar adelante estas metodologías innovadoras necesitan de una importante inversión de tiempo inicialmente en lo relativo a la producción de píldoras audiovisuales y material didáctico para la explicación de la asignatura (Mason, Shuman y Cook, 2013).

Si a esto le sumamos el tiempo que requiere el desarrollo e identificación de problemas o casos prácticos y actividades que deberán realizar los alumnos durante las horas de clase, se concluye que hay que emplearse de forma continuada y contundente. Es lógico que una vez realizado el trabajo de material, éste podrá reutilizarse y aplicarse en futuros cursos académicos, actualizando su contenido de acuerdo a los grupos con los que se trabaja y a las problemáticas que surgieran. 
Unas de las dudas que más se plantean los profesores es saber a qué materia y de qué forma debe iniciarse la aplicación de flipped classroom. En este sentido, la planificación incluirá los principales puntos de partida del aprendizaje, evitando un exceso de detalles que en la primera fase de trabajo no es necesario definir. Antes del tiempo en el aula, el profesor debe tener claras las directrices a plantear y conocer aquello que los alumnos serán capaces de trabajar, hacer y saber desarrollar (Rotellar y Cain, 2016). Dependerá de la creatividad y la originalidad con la que el docente plantee la clase, además de los recursos materiales y virtuales con los que se cuenten. Es fundamental retroalimentar e interrelacionar las tareas que se dan a los alumnos con las actividades a desarrollar en el aula.

Numerosos autores hablan del desafío que supone para el profesor que decide poner en marcha esta metodología y de la necesidad del desarrollo de habilidades en el momento de enfrentarse a recursos multimedia, tecnológicos y otros materiales necesarios en la comunicación y transferencia de la información al alumnado (Ni, Zhen, Xie, Long, Zheng y Li, 2015).

Existen recomendaciones a la hora de realizar la producción del material, la mayoría de ellos vídeos. Estos deben ser concretos, concisos, de alta calidad y de corta duración para captar la atención del alumno, de modo que pueda verlos cuantas veces lo necesite. Lo ideal es que trate un tema o concepto por cada vídeo que se prepara. La inclusión de teorías cognitivas relativas al aprendizaje multimedia proporciona pautas de apoyo de formato práctico a la enseñanza multimedia. Potenciar al máximo las herramientas, prestando especial cuidado en el contenido son las premisas de los expertos. La eliminación de contenidos superficiales y confusos, excluyendo el material redundante y potenciando palabras, gráficos e imágenes con toque personal ayudarán en la creación del material.

Por otro lado, el tiempo de clase al ser tan valioso deberá ser destinado con suma prudencia no solo a intercambiar sobre los contenidos previamente visualizados, sino que deberá propiciar la construcción de razonamientos, reflexiones, pensamientos y conocimientos prácticos, según la disciplina de la que se trate (Rotellar y Cain, 2016)

En todo momento el profesor controla el aula, sirviendo de guía y apoyo a los alumnos, actuando de forma personalizada y conduciendo el aprendizaje hacia los objetivos planteados. Su rol debe ser claro, para controlar y profundizar en determinados puntos de la asignatura que requieran de mayor desarrollo. Es necesario que los alumnos comprendan que su figura es imprescindible, evitando el pensamiento 
que las clases solo consisten en la proyección de vídeos u otros materiales sin la intervención del profesor. Los alumnos deben seguir valorando la presencia y figura del profesor que sin duda aporta un valor añadido (Touchton, 2015).

Tourón y Santiago (2015) describen los roles que deben asumir el profesorado y alumnado comparando un aula tradicional y flipped classroom (tabla 1).

Tabla 1. Roles del docente y alumno en los modelos tradicional y flipped classroom

\begin{tabular}{|c|c|c|c|}
\hline \multirow{2}{*}{ Modelo } & \multirow{2}{*}{ Sujeto } & \multicolumn{2}{|c|}{ Clase } \\
\hline & & Dentro & Fuera \\
\hline \multirow[t]{2}{*}{ Tradicional } & Docente & $\begin{array}{l}\text { Expone los contenidos de forma } \\
\text { presencial. }\end{array}$ & $\begin{array}{l}\text { Envía a los alumnos la realización } \\
\text { de tareas para afianzar los } \\
\text { contenidos aprendidos en clase. }\end{array}$ \\
\hline & Alumno & $\begin{array}{l}\text { Recoge y asimila la información } \\
\text { comunicada por el docente. }\end{array}$ & $\begin{array}{l}\text { Mediante actividades prácticas } \\
\text { propuestas por el docente asimila } \\
\text { los conceptos. }\end{array}$ \\
\hline \multirow[t]{2}{*}{$\begin{array}{l}\text { Flipped } \\
\text { Classroom }\end{array}$} & Docente & $\begin{array}{l}\text { Guía y resuelve dudas. } \\
\text { Profundiza en temas del } \\
\text { aprendizaje. }\end{array}$ & $\begin{array}{l}\text { Expone los contenidos de forma } \\
\text { no presencial, vía multimedia y/o } \\
\text { material didáctico. }\end{array}$ \\
\hline & Alumno & $\begin{array}{l}\text { Desarrolla actividades de } \\
\text { aprendizaje } \\
\text { Socializa con su grupo la } \\
\text { experiencia de aprender. }\end{array}$ & Estudia y asimila los contenidos. \\
\hline
\end{tabular}

Fuente: Tourón y Santiago (2015)

Aunque los aspectos positivos son muchos, también encontramos algunos que son considerados negativos dentro de este método como son los espacios físicos que en su mayoría siguen estructuras de organización clásicas y obstaculizan la organización e intercambio entre los propios alumnos. El mobiliario fijo, la iluminación, las tarimas y las pizarras impiden avanzar en el sentido práctico del método, sabiendo la dificultad que supone tanto para el profesor a la hora de acceder a alumnos que se encuentran en un determinado punto de la clase. Esto influye en el rendimiento y motivación de los alumnos ya que el entorno físico termina por definir la percepción que se tiene de la propia asignatura y su aprendizaje.

\section{Estudios y experiencias de la flipped classroom en la educación superior: revisión de la literatura}

Se ha realizado una revisión bibliográfica sistematizada con objeto de identificar los aspectos más relevantes en diferentes estudios para obtener información sobre las aplicaciones del modelo flipped classroom en la enseñanza universitaria. 
El criterio de selección de artículos de investigación validados ha sido el de palabras clave (universidad, flipped classroom, aprendizaje), en el período de 2013 a 2018 (figura 4). La amplitud del horizonte temporal elegido permite valorar el impacto de la aplicación del modelo en la etapa superior de la enseñanza. Establecidos los criterios de inclusión de los documentos, se procedió a realizar un cribado por título y resumen. A partir de la lectura del texto completo de las referencias, se valoró la relevancia del documento en relación con el interés del estudio. Así, la finalidad es conocer determinadas experiencias relevantes de flipped classrrom en instituciones de educación superior. Del mismo modo, la razón principal de este procedimiento es comparar la relevancia de la información que se obtiene a través de los resultados de la puesta en práctica del modelo pedagógico de la clase invertida. Esta revisión bibliográfica señala puntos congruentes de las características intrínsecas del modelo, como las posibles contradicciones que genera su conceptualización. En los últimos años, numerosos trabajos han estudiado los aspectos más relevantes del modelo de clase invertida (Kim, Kim, Khera y Getman, 2014; Herreid y Schiller, 2013), demostrándose así que su aplicación está adquiriendo importancia en el sistema universitario, en línea con el desarrollo competencial en el EEES.

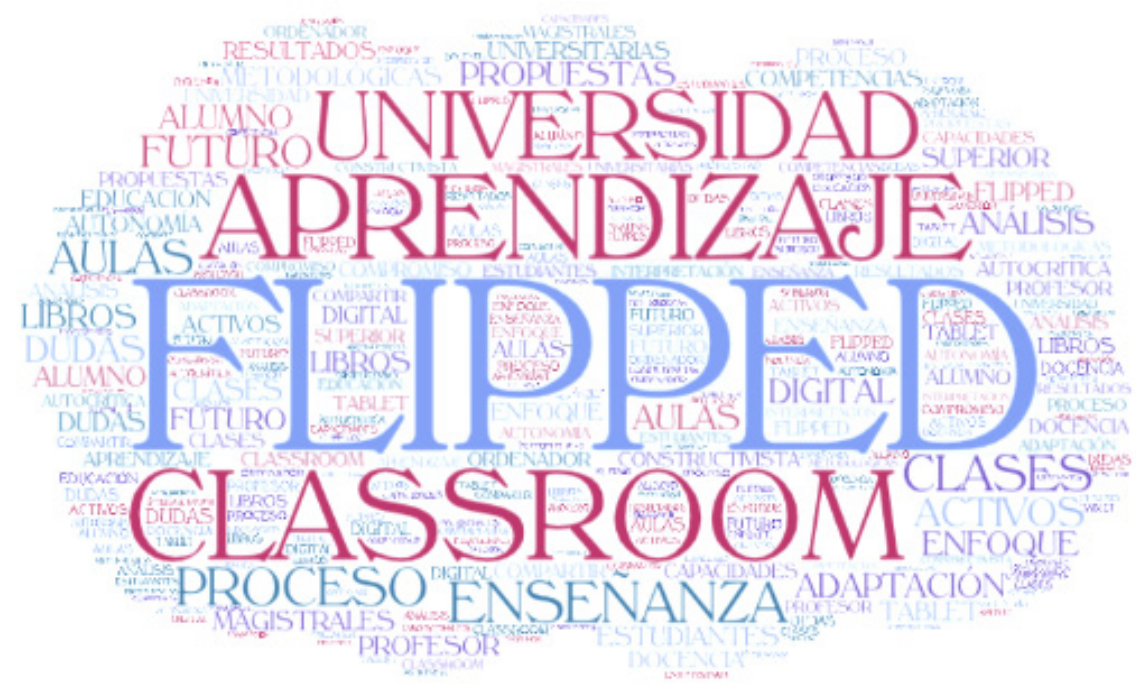

Figura 4. Nube de palabras de las principales palabras clave de la Educación Superior a través de flipped classroom.

En la tabla 2 se muestran los principales resultados obtenidos, tras la puesta en práctica de flipped classroom en la educación superior. Los resultados son positivos, donde se observa, en general, una mayor motivación del estudiante y un incremento en el aprendizaje de los contenidos. 
Tabla 2. Experiencias con flipped classroom en la enseñanza universitaria

\begin{tabular}{|c|c|c|c|}
\hline Institución & $\begin{array}{l}\text { Ciudad } \\
\text { (país) }\end{array}$ & $\begin{array}{c}\text { Referencia } \\
\text { bibliográfica }\end{array}$ & Conclusiones \\
\hline $\begin{array}{l}\text { Universidad Católica de } \\
\text { Valencia 'San Vicente } \\
\text { Mártir' }\end{array}$ & $\begin{array}{l}\text { Valencia } \\
\text { (España) }\end{array}$ & $\begin{array}{l}\text { Angelini, M. L. y } \\
\text { García-Carbonell, A. } \\
\text { (2019) }\end{array}$ & $\begin{array}{l}\text { Los estudiantes que recibieron } \\
\text { instrucción basada en simulación } \\
\text { (grupo experimental) mejoraron } \\
\text { significativamente sus habilidades de } \\
\text { escritura en inglés (en términos de } \\
\text { organización y vinculación de ideas) } \\
\text { más que los estudiantes que asistieron } \\
\text { un curso regular de inglés (grupo de } \\
\text { control) }\end{array}$ \\
\hline Lingnan University & $\begin{array}{l}\text { Tuen } \\
\text { Mun, } \\
\text { Hong } \\
\text { Kong } \\
\text { (China) }\end{array}$ & Ng, E.M. (2018) & $\begin{array}{l}\text { Proporcionó vías para una mayor } \\
\text { interacción durante las lecciones en el } \\
\text { aula. } \\
\text { Permitió una mayor interacción entre } \\
\text { los estudiantes y entre estudiantes- } \\
\text { docentes. }\end{array}$ \\
\hline $\begin{array}{l}\text { Escuela de Ingeniería y } \\
\text { Arquitectura } \\
\text { Facultad de Educación } \\
\text { (Universidad de Zaragoza) }\end{array}$ & $\begin{array}{l}\text { Zaragoza } \\
\text { (España) }\end{array}$ & $\begin{array}{l}\text { Artal, J.S., } \\
\text { Casanova, O., } \\
\text { Serrano, R. y } \\
\text { Romero, E. (2017) }\end{array}$ & $\begin{array}{l}\text { La introducción de dispositivos móviles } \\
\text { en el aula supone sesiones lectivas } \\
\text { interactivas y la participación activa de } \\
\text { los estudiantes. }\end{array}$ \\
\hline $\begin{array}{l}\text { Department of Education } \\
\text { (National Taiwan Normal } \\
\text { University) }\end{array}$ & Taiwan & Hao, Y. (2016) & $\begin{array}{l}\text { En las calificaciones de los cursos de } \\
\text { pregrado, "Information Technology and } \\
\text { Education" y "Classroom Observation, } \\
\text { la preparación para el aprendizaje } \\
\text { autodirigido y las preferencias de trabajo } \\
\text { en grupo pueden predecir las diferentes } \\
\text { dimensiones de la preparación del } \\
\text { Flipped classroom. }\end{array}$ \\
\hline $\begin{array}{l}\text { Universidad Católica de } \\
\text { Valencia 'San Vicente } \\
\text { Mártir' }\end{array}$ & $\begin{array}{l}\text { Valencia } \\
\text { (España) }\end{array}$ & $\begin{array}{l}\text { Angelini, M.L. y } \\
\text { García-Carbonell, A. } \\
\text { (2015) }\end{array}$ & $\begin{array}{l}\text { El alumno desarrolla el trabajo } \\
\text { autónomo, y al mismo tiempo potencia } \\
\text { el trabajo en grupo y colaborativo. }\end{array}$ \\
\hline $\begin{array}{l}\text { Escola Tècnica Superior } \\
\text { Superior d'Enginyeria } \\
\text { Informàtica (ETSINF) } \\
\text { (Universitat Politècnica de } \\
\text { València) }\end{array}$ & $\begin{array}{l}\text { Valencia } \\
\text { (España) }\end{array}$ & $\begin{array}{l}\text { Lluch, C.J., Peñalver, } \\
\text { M.J.P. y Codesal, } \\
\text { E.S. (2014). }\end{array}$ & $\begin{array}{l}\text { En la asignatura "Matemática Discreta", } \\
\text { Flipped classroom presenta ventajas } \\
\text { (aprendizaje más profundo, adquisición } \\
\text { de competencias transversales y } \\
\text { motivación del alumno en el aula), } \\
\text { aunque también presenta aspectos que } \\
\text { pueden dificultar su implementación } \\
\text { (trabajo previo y planificación necesaria } \\
\text { por parte del profesor y no ser siempre } \\
\text { bien aceptada por los estudiantes). }\end{array}$ \\
\hline
\end{tabular}




\begin{tabular}{|c|c|c|c|}
\hline Institución & $\begin{array}{l}\text { Ciudad } \\
\text { (país) }\end{array}$ & $\begin{array}{c}\text { Referencia } \\
\text { bibliográfica }\end{array}$ & Conclusiones \\
\hline $\begin{array}{l}\text { Department of } \\
\text { Curriculum, Culture, and } \\
\text { Educational Inquiry } \\
\text { (Florida Atlantic } \\
\text { University) }\end{array}$ & $\begin{array}{l}\text { Florida } \\
\text { (EEUU) }\end{array}$ & Vaughan, M. (2014) & $\begin{array}{l}\text { Con la lectura tradicional que se } \\
\text { produce fuera del aula, el desafío se } \\
\text { convierte en cómo maximizar el tiempo } \\
\text { de aprendizaje adicional. } \\
\text { Así, la formación del docente en los } \\
\text { distintos métodos educativos, pueden } \\
\text { marcar una diferencia significativa en el } \\
\text { aprendizaje de los alumnos. }\end{array}$ \\
\hline $\begin{array}{l}\text { Departamento de } \\
\text { Ingeniería Mecánica } \\
\text { (Seattle University) }\end{array}$ & $\begin{array}{l}\text { Seattle } \\
\text { (EE.UU) }\end{array}$ & $\begin{array}{l}\text { Mason, G.S., } \\
\text { Shuman, T.R. y } \\
\text { Cook, K.E. (2013) }\end{array}$ & $\begin{array}{l}\text { Proporcionó que el tiempo de clase se } \\
\text { dedicara a la resolución de problemas } \\
\text { individuales y grupales. } \\
\text { El docente pudo aportar más materia. } \\
\text { Los estudiantes obtuvieron mejores } \\
\text { calificaciones en los exámenes. }\end{array}$ \\
\hline
\end{tabular}

En las últimas décadas, esta metodología ha contribuido a la revisión del conocimiento científico, utilizándose para estudiar un determinado campo de investigación. Así, gracias a esta herramienta, se puede mostrar la información bibliográfica de las principales tendencias de investigación de las experiencias a través de flipped classroom en las instituciones de educación superior. La aplicación de un enfoque competencial en la etapa de la educación superior, está en línea con el modelo pedagógico, en el sentido de formar a ciudadanos que sean capaces de adaptarse a las características de una sociedad cambiante, donde la reflexión de los acontecimientos será clave en un entorno globalizado.

\section{Conclusiones}

Las exigencias que impone el EEES se dirigen a cambios sustanciales y el planteamiento de nuevos modelos educativos que favorezcan una enseñanza-aprendizaje más dinámica basada en aprendizajes autónomos, donde se fomente la práctica, el trabajo colaborativo y el uso de materiales y técnicas adaptables.

La interacción y aprovechamiento máximo de su aprendizaje viene dado por el menor número de alumnos. De esta manera, aportar conocimiento al grupo genera un beneficio común, modificando el concepto individualista por la búsqueda del beneficio grupal. 
La lección magistral no debe desaparecer, sobre todo en los primeros cursos universitarios donde el alumno está en una etapa de adaptación y cuando mayor es la carga de contenidos teóricos. Aun así, en esta primera etapa de la enseñanza superior se deberán combinar en todas las asignaturas la lección magistral con métodos más activos, para que el alumno adquiera las competencias que definen a los nuevos modelos pedagógicos.

Flipped classroom es un modelo educativo que contribuye a mejorar la educación superior, siendo fundamental trabajar en su correcto diseño para obtener los resultados deseados. Su aplicación proporciona protagonismo al alumno desarrollando mediante el trabajo en equipo estrategias de asimilación de conceptos, aportando en el estudiante autonomía, reflexión y estimulación.

El diseño de los contenidos, tarea a cargo del profesor, deberá hacer hincapié en el uso de las herramientas adecuadas para desarrollar la calidad y comprensión de los contenidos a impartir.

El rol del profesor universitario deja de ser la tradicional, es decir, pasa de ser un transmisor de conocimientos a transformarse en guía y orientador del aprendizaje académico. En este camino, es necesario que toda la comunidad universitaria, reaccione y participe a los cambios que el EEES reclama y exige.

La motivación de cambio por parte del profesorado necesita del apoyo del alumno. A él habrá que reeducarlo de forma activa y participativa frente al nuevo escenario planteado. Será su obligación la de actuar frente a los nuevos estímulos propuestos.

La presencia de las nuevas tecnologías contribuye a que el diálogo profesor-alumno sea más próximo, fomentando el uso de sus herramientas digitales que permiten progresar en nuevas formas de enseñar y aprender. Dejar de lado los conceptos reiterativos de las clases magistrales, permiten ampliar las posibilidades del acto académico.

Se comprueba que la clase invertida proporciona la adquisición de competencias específicas y transversales en los estudiantes de instituciones de enseñanza superior, mejorando así su competitividad. Así, se observa que este está en línea con el sistema de transferencia de créditos (sistema ECTS), donde la metodología docente implica un papel más activo por parte del estudiante que con la tradicional lección magistral. En numerosos estudios se concluye que tanto el docente como el alumno muestran resultados satisfactorios, aunque siempre dependerá de una aplicación concisa y bien estructurada. 


\section{Referencias bibliográficas}

Abeysekera, L. y Dawson, P. (2015). Motivation and cognitive load in the flipped classroom: definition, rationale and a call for research. Higher Education Research \& Development, 34(1), 1-14. https://doi.org/10.1080/07294360.2014.934336

Acaso López-Bosch, M. (2013). rEDUvolution: hacer la revolución en la educación. Grupo Planeta (GBS).

Andrade, E. y Chacón, E. (2018). Implicaciones teóricas y procedimentales de la clase invertida. Pulso. Revista de Educación, 41, 251-267. Recuperado de https://bit.ly/2Mc5Xvd

Angelini, M.L. y García-Carbonell, A. (2015). Percepciones sobre la integración de modelos pedagógicos en la formación del profesorado: la simulación y juego y el flipped classroom. Education in the Knowledge Society, 16(2), 16-30. Recuperado de: https://bit.ly/2lf85gD

Angelini, M.L. y García-Carbonell, A. (2019). Enhancing students' written production in English through flipped lessons and simulations. International Journal of Educational Technology in Higher Education, 16(1), 2.

Artal, J.S., Casanova, O., Serrano, R. y Romero, E. (2017). Dispositivos móviles y Flipped Classroom. Una experiencia multidisciplinar del profesorado universitario. Edutec. Revista Electrónica de Tecnología Educativa, 59, 355. https://doi.org/0.21556/ edutec.2017.59.817.

Bergmann, J. y Sams, A. (2012). Flip your classroom: Reach every student in every class every day. Washington, DC: International society for technology in education/ISTE.

Bergmann J. y Sams A. (2014). Dale la vuelta a tu clase. Madrid: Editorial SM.

Cabrera, V.A. (2005). El concepto calidad en la educación universitaria: clave para el logro de la competitividad institucional. Revista iberoamericana de Educación, 36(12), 1-7.

Díaz-Barriga, Á. (2011). Competencias en educación. Corrientes de pensamiento e implicaciones para el currículo y el trabajo en el aula. Revista Iberoamericana de Educación Superior, II (5), 3-24.

García, R., Traver, J. y Candela, I., (2001). Aprendizaje cooperativo. Fundamentos, características y técnicas. Madrid: CCS.

Gutiérrez Martén, A., Palacios Picos, A. y Torrego Egido, L. (2010). Tribus digitales en las aulas universitarias. Comunicar, 17(34), 173-181.

Hamdan, N., Mcknight, P., Mcknight, K. y Arfstrom, K.M. (2013). A Review of Flipped Learning. Flipped Learning Network, 1-4.

Hao, Y. (2016). Exploring undergraduates' perspectives and flipped learning readiness in their flipped classrooms. Computers in Human Behavior, 59, 82-92. https://doi. org/10.1016/j.chb.2016.01.032.

Herreid, C.F. y Schiller, N.A. (2013). Case studies and the flipped classroom. Journal of College Science Teaching, 42(5), 62-66. 
Huber, G.L. (2008). Aprendizaje activo y metodologías educativas. Revista de Educación, $59,59-81$.

Johnson, D.V y Johnson, R. (1991). Learning together and alone. Cooperative, competitive and individualistic learning. Needham Heights, Allyn and Bacon.

Kim, M.K., Kim, S.M., Khera, O. y Getman, J. (2014). The experience of three flipped classrooms in an urban university: an exploration of design principles. The Internet and Higher Education, 22, 37-50.

Lluch, C.J., Peñalver, M.J.P. y Codesal, E.S. (2014). Investigación del impacto en un aula de matemáticas al utilizar flip education. In Pensamiento matemático, Universidad Politécnica de Madrid, 4(2), 9-22.

Kagan, S. (1994). Cooperative Learning. San Clemente, CA: Kagan.

Lage, M.J., Platt, G.J. y Treglia, M. (2000). Inverting the classroom: A gateway to creating an inclusive learning environment. The Journal of Economic Education, 31(1), 30-43.

Manresa, S.T. (2018). Flipped Classroom: Un modelo pedagógico eficaz en el aprendizaje de Science. Revista Iberoamericana de Educación, 76(1), 9-22. Recuperado de https://doi.org/10.35362/rie7612969

Mason, G.S., Shuman, T.R. y Cook, K.E. (2013). Comparing the Effectiveness of an Inverted Classroom to a Traditional Classroom in an Upper-Division Engineering Course. IEEE Transactions on Education, 56(4), 430-435.

Mok, H.N. (2014). Teaching tip: The flipped classroom. Journal of Information Systems Education, 25(1), 7.

Ng, E.M. (2018). Integrating self-regulation principles with flipped classroom pedagogy for first year university students. Computers \& Education, 126, 65-74.

Ni, M., Zhen, L., Xie, Y., Long, H., Zheng, X. y Li, W. (2015). A study of an e-schoolbag supporting flipped classroom model for junior mathematics review class. In International Conference on Hybrid Learning and Continuing Education (243254). Springer, Cham.

Phillips, C.R. y Trainor, J.E. (2014). Millennial students and the flipped classroom. Proceedings of ASBBS, 21(1), 519-530.

Prieto, L. (2007a). El aprendizaje cooperativo. Madrid: PPC.

Prieto, L. (2007b). La enseñanza universitaria centrada en el aprendizaje. Barcelona: Octaedro/ICE UB.

Rotellar, C. y Cain, J. (2016). Research, Perspectives, and Recommendations on Implementing the Flipped Classroom. American Journal of Pharmaceutical Education, 80(2), 34.

Sánchez, V.G. y Arrufat, M.J.G. (2016). Modelo de análisis de metodologías didácticas semipresenciales en Educación Superior. Educación XX1, 19(1). https://doi. org/10.5944/educxx1.15577 
Thai, N.TT., De Wever, B. y Valcke, M. (2017). The impact of a flipped classroom design on learning performance in higher education: Looking for the best "blend" of lectures and guiding questions with feedback. Computers \& Education, 107, 113-126. https://doi.org/10.1016/j.compedu.2017.01.003

Touchton, M. (2015). Flipping the Classroom and Student Performance in Advanced Statistics: Evidence from a Quasi-Experiment. Journal of Political Science Education, 11(1), 28-44. https://doi.org/10.1080/15512169.2014.985105

Tourón, J. y Santiago, R. (2015). El modelo Flipped Learning y el desarrollo del talento en la escuela. Revista de Educación, 368, 196-231. Recuperado de https://bit. $\underline{\mathrm{ly} / 1 \mathrm{dHH} 8 \mathrm{~K} 7}$

Vaughan, M. (2014). Flipping the learning: An investigation into the use of the flipped classroom model in an introductory teaching course. Education Research and Perspectives, 41, 25-41. Recuperado de https://bit.ly/2KeJSt4

Zañartu, L.M. (2003). Aprendizaje colaborativo: una nueva forma de diálogo interpersonal y en red. Contexto Educativo, 28. 